\title{
A generalized strip deflexion method of reinforced concrete slab design
}

\author{
J. S. FERNANDO \& K. O. KEMP
}

\section{Professor P. G. Lowe, University of Strathclyde}

The Authors have outlined an interesting extension to the strip method of slab design which promises more economical designs as well as scope to deal with difficult cases. Besides the comparisons made in the Paper it is perhaps appropriate to seek the comparative moment volumes for the problem posed by the Authors when looked at from the strip, minimum weight and yield line viewpoints.

47. For the problem posed of side ratio 1.4 and the simply supported pair of edges in conjunction with a fixed and a free edge, optimum theory ${ }^{7}$ gives the minimum weight moment volume to be $V_{0}=0 \cdot 115 p L^{4}$. A yield line solution, isotropic for bottom steel and using top steel in one direction only extending to $L / 4$ from the fixed edge, gives $V_{\mathrm{yL}}=0 \cdot 260 p L^{4}$. The Authors' proposals for a strip solution give $V_{\mathrm{s}}=0 \cdot 150 p L^{4}$.

48. An extreme form of strip solution in which $q_{x}=0$ in a uniform strip bordering the fixed edge and $q_{y}=0$ in the remainder of the slab area can be shown to produce a minimum moment volume of $V_{1}=0 \cdot 141 p L^{4}$ when the $q_{x}=0$ region occupies $57 \%$ of the slab area. Such a solution is more economical than the Authors' proposal, although is no doubt less satisfactory in practical terms due to the disregard for serviceability considerations.

49. The conclusion seems to be that the strip method bears a roughly similar relation to the minimum weight solution for this problem, as was the case for the square simply supported slab (Table 1). However, the yield line solution appears to be significantly less economical in this case when compared with the minimum weight solution.

50. The Authors draw attention to the special role of solutions in which the principal moments are locally of the same sign. Both the solutions labelled $V_{0}$ and $V_{1}$ above are of this type. According to minimum weight theory ${ }^{8}$ such cases imply that bending moments deriving from the isotropic elastic solutions (with Poisson's ratio zero) are permissible minimum weight bending moment fields. However, such bending moment fields are usually not the only fields which minimize the moment volume. This observation would seem to account for the remarkable agreement between Wood' $s^{9}$ numerical calculation of moment volume for the simply supported square slab $\left(V_{\theta}=\right.$ $0.052 p L^{4}$ ) and the later derived minimum weight value of $V_{0}=5 p L^{4} / 96=0.052 p L^{4}$.

51. This observation too would seem to support the view that at least such minimum weight solutions are likely to meet serviceability criteria in so far that they have a good deal in common with an elastic field of bending moments.

Dr L. A. Clark, Department of Civil Engineering. University of Birmingham

In $\$ 4$ the Authors state that the proposed strip deflexion method can be extended to accommodate concentrated loads and supports, but this aspect of the method is not 


\section{DISCUSSION}

developed in the Paper. Are there any problems, of which the designer should be aware, associated with such an extension to the method?

53. The Authors relate their method to grillage analysis using orthogonal members; have they also examined skew grillages in this context?

\section{Dr P. Bhatt, Glasgow University}

The Authors recommend that the moment field for the designing of the slabs by the strip method should be obtained by considering the compatibility of deflexions at the strip intersection points. This can be achieved either by the strip deflexion method or by treating the slab as a torsionless grid. By such an analysis it is expected that the resulting moment field will not be too different from the true elastic field.

55. Figures 5(a) and 5(b) show the design moment fields $M^{*}$ and $M^{*}$ for a simply supported square slab of $3 \mathrm{~m}$ sides and carrying an ultimate uniformly distributed load of $10 \mathrm{kN} / \mathrm{m}^{2}$ calculated on the basis of elastic plate analysis, including torsion, and strip analysis using the load distribution factors shown. The elastic plate analysis was done using the Fourier series solution to obtain $M_{x}, M_{y}$ and $M_{x y}$. Poisson's ratio was taken to be $0 \cdot 2$. The design moments $M^{*}$ x and $\dot{M}^{*}{ }_{y}$ were calculated using the equations developed by Wood ${ }^{10}$ based on Johansen's stepped yield criterion. The load distribution factors were not very different from the values obtained from strip deflexion analysis. The conclusion from the comparison is that the design moments given by the two me-

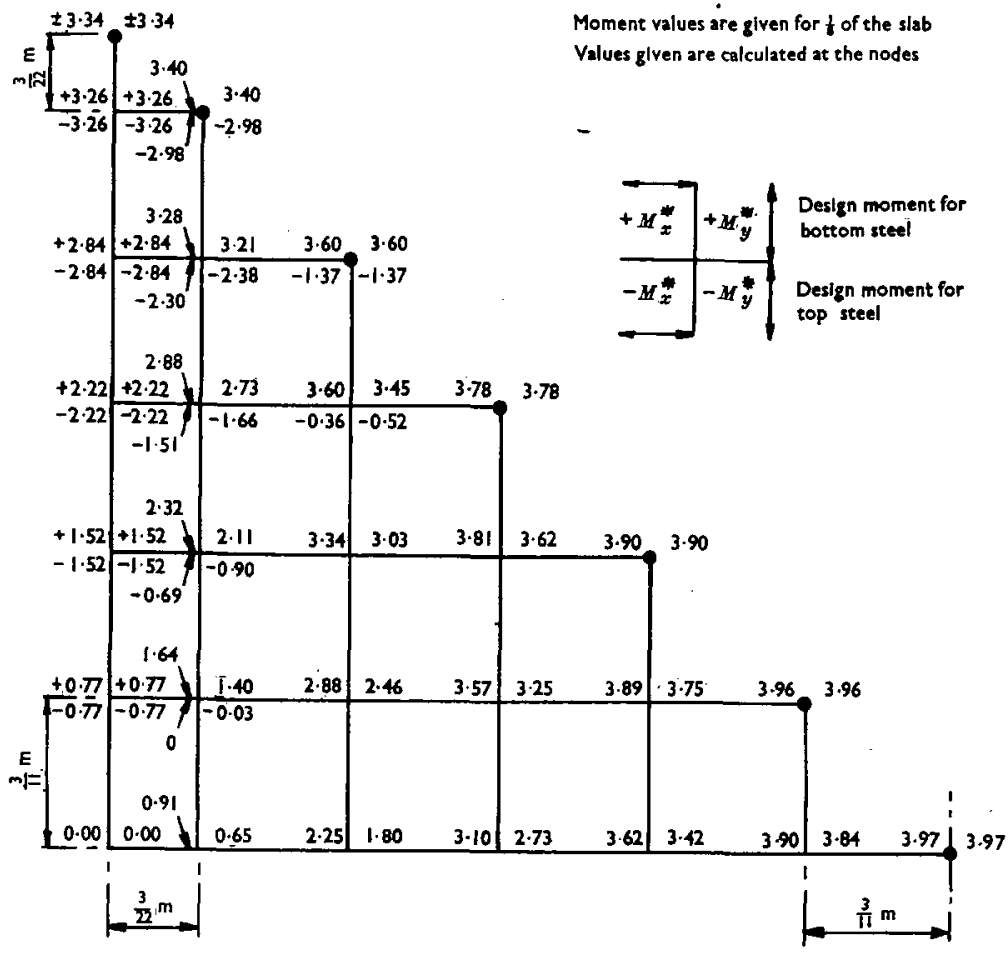

Fig. 5(a). Design moments in $\mathrm{kNm} / \mathrm{m}$ for an elastic slab using Wood's equations ${ }^{10}$ 
thods are very different. Presumably when designing the slabs by the strip method one adds to the calculated reinforcement additional torsion steel as given in CP $110 .^{5}$

56. I feel that the only reason for neglecting torsion as recommended by Hillerborg ${ }^{1}$ is because it leads to a simple procedure for hand calculations. The main disadvantage is that it is difficult to decide the appropriate load distribution factors without jeopardizing the behaviour at working loads. If one has to use a computer program to analyse the slab, the best procedure is to include the torsion as well, whether the analysis is done by the finite element method or by the grid analogy method.

Dr R. E. Melchers, Department of Civil Engineering, Monash University, Australia

The Authors justify their method on the grounds of two 'disadvantages': the high degree of freedom available to designers in selecting load distributions and the resultant danger of poor cracking behaviour at working load, and because the simple method breaks down with concentrated loads and supports. Unfortunately, they offer no real evidence that the strip method does indeed produce unsatisfactory behaviour when used with the degree of structural understanding one would normally expect from a competent structural designer. In fact, there is (admittedly limited) experimental evidence to suggest that for simply supported slabs the method produces designs of acceptable behaviour. Hillerborg ${ }^{1}$ reviewed the published literature to about 1973.

58. I have tested square slabs with clamped edges, but unrestrained in the plane of the

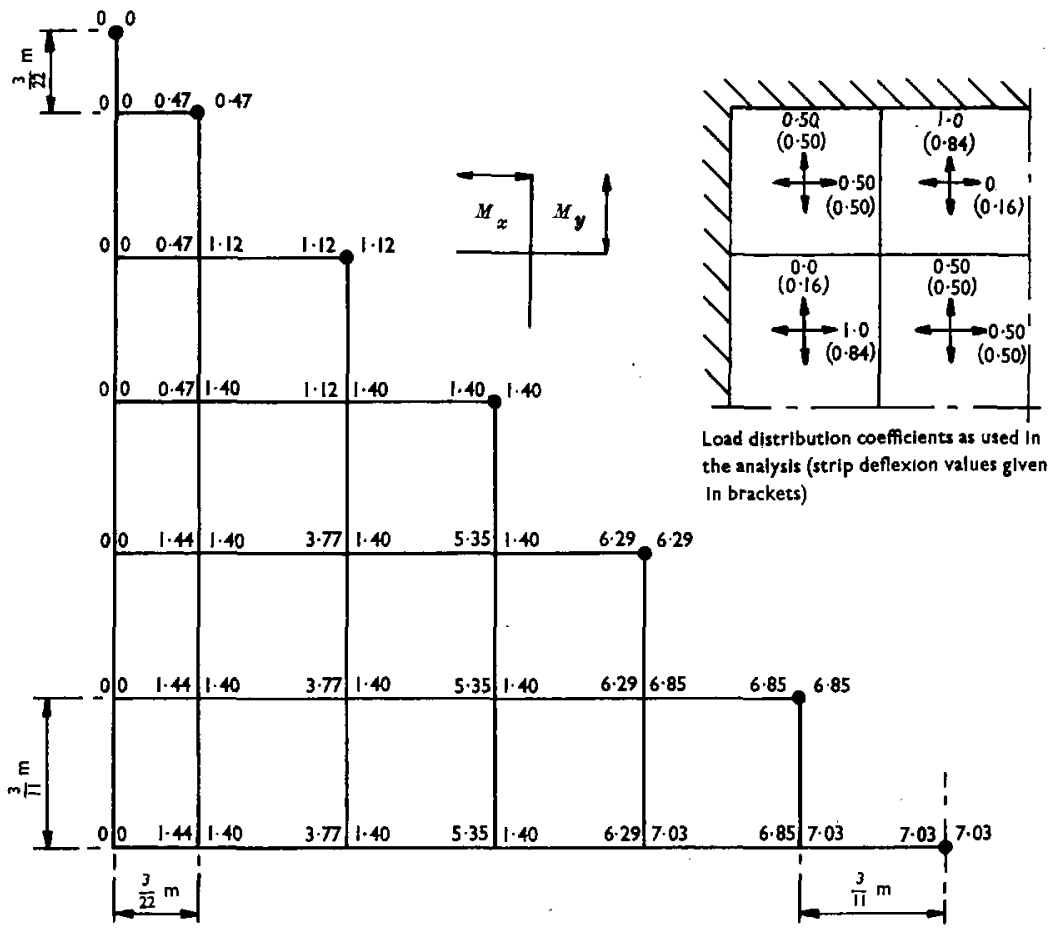

Fig. 5(b). Design moments in $\mathrm{kNm} / \mathrm{m}$ for an elastic slab using strip method 
slab, having reinforcement proportioned by minimal reinforcement criteria. Such reinforcement is based on a moment field in equilibrium with the applied design load, but departs dramatically from conventional layouts such as the square mesh commonly used for yield line analysis or rectilinear patterns for code-based design. Thus, although the layout of reinforcement is based on optimality criteria, the requirements of equilibrium and of the yield condition are satisfied, so that the optimal designs are also Hillerborg designs. The tests show that the behaviour in terms of cracking and deflexion, as well as the load capacity, was satisfactory, even at relatively low loads, when compared with tests on slabs designed according to yield line theory. These tests therefore lend support to the results of earlier tests that quite large deviations from elastic theory can be tolerated in the design of reinforced concrete slabs when proportioning slab reinforcement.

59. By introducing the compatibility of deflexion criteria, the Authors have removed the great (apparent) freedom available to designers and in so doing have probably made the method more fool-proof for novice designers, but only at the expense of considerable additional complexity. In view of the test results, it is likely that the better behaviour the Authors clearly expect using the strip deflexion method is probably more imaginary than real. The fundamental question is really how well the linear elastic solution refiects slab behaviour in terms of concrete cracking.

60. The Authors show how their method works for square and rectangular slabs. While the simple strip method is easily extended to irregular slab plan shapes, is this also true for the Authors' method?

61. Concentrated loads and supports offer problems for all slab design procedures. Hillerborg's advanced method is clearly too complex for normal use. However, the procedure for spreading a line load over an area of slab (Hillerborg, ${ }^{1}$ p. 57 ) can be adapted easily for use with point loads (or reactions) through the use of stress dispersion in the thickness of the slab, and thence through the use of orthogonal concentrated bands of reinforcement to produce an equivalent distributed load on the slab.

62. The Authors give no details as to how the strip deflexion method can handle point loads, despite their criticism of the normal strip method on this point. Could they indicate their procedure?

\section{Dr J. Harrop, Department of Civil Engineering, University of Leeds}

As the Authors point out, because of the freedom that designers have in using the strip method in the choice of load dispersion systems, a check on serviceability conditions becomes particularly important. The strip deflexion method, using twistless elastic moment fields, gives a measure of control in this respect. However, significant torsional moments may exist in many slab configurations at working load conditions and comparisons of twistless moment fields with moment fields including twists would be of interest. Have the Authors compared the moment fields obtained from their strip deflexion method with moment fields where torsional moments are included ?

64. The fact that the strip deflexion gives twistless moment fields may well make it particularly appropriate for the design of slabs of the waffle, or two-way ribbed, type because the torsional stiffness of such slabs is lower than that of solid slabs. Tebbett ${ }^{11}$ has shown that in a ribbed slab little load is carried by twisting action.

65. For rectangular slabs simply supported on beams the Rankine-Grashof $\left(L_{y} / L_{x}\right)^{4}$ load distribution rule will give an exact elastic twistless system of moments provided certain specific stiffnesses for the supporting beams are used. It can be shown, from the work of Wood, ${ }^{8}$ that the ratios of the elastic stiffnesses of the supporting beams to the adjoining slab half widths have to be

$$
\begin{gathered}
\gamma x=2 E I_{x} / D L_{y}=\left(L_{x} / L_{y}\right)^{4} \\
\gamma y=2 E I_{y} / D L_{x}=\left(L_{y} / L_{x}\right)^{4} \\
I_{y} / I_{x}=\left(L_{y} / L_{x}\right)^{7}
\end{gathered}
$$


Elastic twistless solutions also exist for load distributions that are not according to the $\left(L_{y} / L_{x}\right)^{4}$ rule; the condition then is that $\gamma x \gamma y=1$. For such solutions the distribution of the bending moments across the slabs in the two orthogonal directions is uniform, as is the load distribution on the supporting beams. These elastic twistless solutions provide useful landmarks in the study of slab-beam systems.

66. In the context of beam supported slabs the search for solutions that give minimum quantities of reinforcement in the slab alone is to a large extent meaningless because any attempt to do this will simply result in more moment being directed into the supporting beams with consequent increase in reinforcement quantities there. ${ }^{12}$ It should be remembered that for an orthogonal system of slabs and beams the total equilibrium bending moments must be carried by the beams and slab combined in each of the two orthogonal directions. It is therefore better from a practical point of view to use relatively simple banded systems for the slab reinforcement. For continuous slabs the question of reinforcement minimization is bound up with the ratio of the continuity moment to the span moment in each strip.

\section{Dr Fernando and Professor Kemp}

Both Professor Lowe and Dr Melchers stress the advantages of optimal solutions, arguing that reinforced concrete slabs can tolerate quite large deviations from elastic theory when proportioning the reinforcement. In contrast, Dr Bhatt and Dr Harrop seem concerned that the twisting moments are omitted and the strip deflexion solutions may depart too far from elastic conditions. We agree with Dr Harrop when he suggests that simple banded layouts of reinforcement are more desirable for economic reasons than absolute minimum weights of reinforcement.

68. The omission of twisting moments is, however, an essential feature of the strip method, because the orthogonal reinforcement directions then become principal moment directions and exact solutions for the collapse loads will be obtained. Including the twisting moments merely increases the complexity of the calculations to produce a lower bound solution. The same argument applies to the question of using skew strips raised by $\mathrm{Dr}$ Clark as this would also involve twisting moments and loss of uniqueness of the solutions.

69. Dr Melchers suggests that there are no problems with the Hillerborg simple strip method when used by competent designers. We would question this observation, particularly when applied to irregular slabs and concentrated loading which have stretched the imagination of even such a gifted designer as Hillerborg. Surely it is desirable to produce methods which are as fool-proof and systematic as possible to protect the less than wholly competent designer in codes of practice recommendations.

70. Dr Bhatt shrewdly compares the design moments for a simply supported square slab obtained from an elastic and an approximate strip deflexion solution. Admittedly, the moment fields are significantly different, but then so are the two slabs because in one case (elastic) the corners are held down and in the other they are not. The worst discrepancy which exists between the strip method and an elastic solution is under pure twist when the strips are chosen in the direction of the axes of twisting moment. The strip solution then gives zero moments and the elastic field $M_{x y}$ in both directions in the top and bottom faces of the slab. To deal properly with pure twist the strips should be chosen in directions at $45^{\circ}$ to the axes of the twisting moments. In Dr Bhatt's elastic solution, which is a safe lower bound solution, it is the pure twist in the restrained corners which causes the significant differences in the moment field, although his approximation of the strip deflexion method loading has worsened the comparison. We would suggest that in applying the strip deflexion method to this problem, the edge strips should be treated as clamped at the corners. The differences between the strip deflexion and elastic design moments would then be much less than in the examples he illustrates. However, it must be emphasized that we do not aim to reproduce the elastic moment fields in the strip deflexion method, but rather to develop solutions which do not depart so far from working load conditions as to affect serviceability, and yet to produce a 
unique solution for the collapse load involving simple banded layouts of reinforcement.

71. On the question of how the strip deflexion method can be extended to accommodate concentrated loads, we assume that all concentrated loads cover a finite area of the slab and if the area is large enough a strip system can be chosen so that there is uniform loading over a grid area. The analysis is then identical to the procedure described in the Paper and load distributions will be obtained for all the grid areas both loaded and unloaded. However, if the concentrated loaded area is small a strip system is chosen so that the concentrated load is centrally placed in a grid area. It is then assumed that the concentrated loading is uniformly dispersed over the whole grid area and the same analysis carried out. To satisfy equilibrium and preserve the uniqueness of the solution, additional reinforcement is required within the grid area carrying the concentrated load. This can be readily calculated using the equilibrium fields first proposed by Hillerborg and referred to by Dr Melchers; the additional moments affect only the loaded grid area and depend only on the load and geometry of the loaded and grid areas so that standard solutions can be determined.

72. Dr Clark asks if any problems arise in the extension of the strip deflexion method to accommodate concentrated loads. Theoretically there are none, but practically there is a need for some large-scale tests to determine the maximum permissible ratio of grid area to concentrated loaded area to maintain adequate serviceability.

73. No problems are known to us for dealing with irregular shaped slabs by the strip deflexion method. If a torsionless grid system can be devised for the problem, which should always be possible for a stable structure, the method is applicable.

74. We were particularly pleased to note Dr Harrop's remarks about the need to consider supporting beams in the design of slab systems. This is an area where present codes of practice seem to be deficient in that only a crude treatment of the loads on supporting beams is presented. One of the advantages of the strip deflexion method is that the load distribution on the supporting beams is fully described and the moments in the slab and beans take account of beam and slab relative flexibilities. This is desirable to ensure adequate serviceability and yet the uniqueness of the collapse loads is preserved.

\section{References}

7. Melchers R. E. and Lowe P. G. Discussion on Rectangular grillages of least weight,-by G. I. N. Rozvany and S. R. Adidan. J. Engng Mech. Div. Am. Soc. Civ. Engrs, 1973, 99, EM6, Dec., 1301-1304.

8. Lowe P. G. and YASSERI S. F. Non-linear optimised fibre reinforced plates in bending. Private communication.

9. Woon R. H. Plastic and elastic design of slabs and plates. Thames and Hudson, London, 1960, 202.

10. Woon R. H. The reinforcement of slabs in accordance with a pre-determined field of moments. Concr., 1968, 2, No. 2, Feb., 69-76.

11. TeBbetT I. E. Analysis and design of rib-stiffened plates and slabs. $\mathrm{PhD}$ thesis, University of Leeds, 1976.

12. Harrop J. Ultimate load design of simple-supported slab-beam systems. Bldg Sci., 1969, 4, 145-150. 